\title{
Revisiting gravitino dark matter in thermal leptogenesis
}

\author{
Masahiro Ibe,,$^{a, b}$ Motoo Suzuki $^{a}$ and Tsutomu T. Yanagida ${ }^{a}$ \\ ${ }^{a}$ Kavli IPMU (WPI), UTIAS, The University of Tokyo, \\ Kashiwa, Chiba 277-8583, Japan \\ ${ }^{b}$ ICRR, The University of Tokyo, \\ Kashiwa, Chiba 277-8582, Japan \\ E-mail: ibe@icrr.u-tokyo.ac.jp, m0t@icrr.u-tokyo.ac.jp, \\ tsutomu.tyanagida@ipmu.jp
}

ABSTRACT: In this paper, we revisit the gravitino dark matter scenario in the presence of the bilinear $R$-parity violating interaction. In particular, we discuss a consistency with the thermal leptogenesis. For a high reheating temperature required for the thermal leptogenesis, the gravitino dark matter tends to be overproduced, which puts a severe upper limit on the gluino mass. As we will show, a large portion of parameter space of the gravitino dark matter scenario has been excluded by combining the constraints from the gravitino abundance and the null results of the searches for the superparticles at the LHC experiments. In particular, the models with the stau (and other charged slepton) NLSP has been almost excluded by the searches for the long-lived charged particles at the LHC unless the required reheating temperature is somewhat lowered by assuming, for example, a degenerated right-handed neutrino mass spectrum.

KeYWORDS: Supersymmetry Phenomenology

ArXiv EPrint: 1609.06834 


\section{Contents}

1 Introduction 1

2 R-parity violation and the gravitino dark matter 2

3 gravitino dark matter in thermal leptogensis scenario 4

3.1 Thermal gravitino production 4

$\begin{array}{lll}3.2 & \text { NLSP contributions } & 6\end{array}$

$\begin{array}{lll}3.3 & \text { Collider constraints } & 7\end{array}$

4 Conclusions and discussions $\quad 11$

A A model of $\boldsymbol{R}$-parity violation $\quad \mathbf{1 2}$

A.1 $R$-parity violation tied with $\mathrm{U}(1)_{B-L}$ breaking $\quad 12$

$\begin{array}{lll}\text { A.2 } & \text { Model with discrete } R \text {-symmetry } & 14\end{array}$

\section{Introduction}

For decades, supersymmetry has been widely studied as one of the top candidates for physics beyond the Standard Model (SM) which allows a vast separation of low energy scales from high energy scales such as the Planck scale. The precise unification of the gauge coupling constants at the scale of the grand unified theory (GUT) also strongly supports the minimal supersymmetric standard model (MSSM). In addition, when the $R$ parity $[1,2]$ is imposed to forbid baryon $(B)$ and lepton $(L)$ number violating interactions, the lightest supersymmetric particle (LSP) becomes a good candidate for dark matter.

Although the $R$-parity is very important phenomenologically, understanding of its origin remains as an open question [3]. In fact, in view of general discussion that all global symmetries are necessarily broken by quantum gravity effects [4-9], the $R$-parity is potentially violated unless it is embedded in gauged symmetries. ${ }^{1}$

A popular framework for such embedding is to identify the $R$-parity (or the matter parity [14-17]) with a discrete $\mathbb{Z}_{2}$ subgroup of the gauged $\mathrm{U}(1)_{B-L}$ symmetry, which naturally emerges once we introduce right-handed neutrinos required for the seesaw mechanism $[18,19]$ [see also 20]. There, the Majorana masses of the right-handed neutrinos are induced when the $\mathrm{U}(1)_{B-L}$ symmetry is broken down to its $\mathbb{Z}_{2}$ subgroup spontaneously.

This framework is, however, known to have a tension with perturbative $\mathrm{SO}(10)$ GUT. There, the Majorana mass terms of the right-handed neutrinos require the vacuum expectation value (VEV) of fields in $\mathbf{1 2 6}$ or larger representations of $\mathrm{SO}(10)$. However, an introduction of fields in such large representations causes a rapid blow up of the $\mathrm{SO}(10)$

\footnotetext{
${ }^{1}$ Discrete subgroups of the gauge symmetries are immune to quantum gravitational effects [10-13].
} 
gauge coupling constant just above the GUT scale. To avoid this problem, it is often assumed that $\mathrm{U}(1)_{B-L}$ by a VEV of $\overline{\mathbf{1 6}}$ representation with which the Majorana masses are given by $\langle\overline{\mathbf{1 6}}\rangle^{2}$. In this case, the $\mathbb{Z}_{2}$ subgroup of $\mathrm{U}(1)_{B-L}$ does not remain unbroken, and hence, no exact $R$-parity remains. ${ }^{2}$ Rather, this argument opens up a new framework $^{3}$ where small $R$-parity violation effects are tied with $\mathrm{U}(1)_{B-L}$ breaking as pursued in refs. [22, 23].

Once $R$-parity violation is accepted, the gravitino LSP has a definite advantage to be a candidate for dark matter. Compared with other LSP candidates, the gravitino LSP can have a much longer lifetime even in the presence of $R$-parity violation [24, 25].

In this paper, we discuss gravitino dark matter in the presence of the $R$-parity violating interactions. In particular, we revisit a consistency with the thermal leptogenesis [26] [see 27-29, for review]. For a high reheating temperature required for the thermal leptogenesis, the gravitino dark matter tends to be overproduced, which puts a severe upper limit on the gluino mass $[22,30,31]$. As we will show, a large portion of parameter space of the gravitino dark matter scenario has been excluded by combining the constraints from the gravitino abundance and the null results of the searches for the superparticles at the LHC experiments.

The organization of this paper is as follows. In section 2, we briefly review $R$-parity violation in the MSSM. We also review the gravitino properties in the presence of $R$-parity violation. In section 3 , we discuss a consistency between the gravitino dark matter scenario and thermal leptogenesis scenario. There, we also discuss the constraints from the LHC experiments. Final section is devoted to our conclusions and discussions.

\section{R-parity violation and the gravitino dark matter}

Let us briefly review $R$-parity violation in the MSSM (see [32] for a detailed review). The general renormalizable $R$-parity violating superpotential is given by

$$
W_{\not K}=\frac{1}{2} \lambda_{i j k} L_{L i} L_{L j} \bar{E}_{R k}+\lambda_{i j k}^{\prime} L_{L i} Q_{L j} \bar{D}_{R k}+\frac{1}{2} \lambda_{i j k}^{\prime \prime} \bar{U}_{R i} \bar{D}_{R j} \bar{D}_{R k}+\mu_{i}^{\prime} L_{L i} H_{u},
$$

where $i, j, k=1,2,3$ denote the family indices of the matter fields. The coefficients $\lambda^{(\prime, \prime \prime)}$ and $\mu^{\prime}$ are dimensionless and dimensionful parameters of $R$-parity violation, respectively. The third term violates the $B$-number while the other terms violate the $L$-number.

The most universal constraints on $R$-parity violation come from cosmology. In the presence of the $B$ and/or $L$-number violating processes induced by $R$-parity violation, the baryon asymmetry generated before the electroweak phase transition would be washed out. To avoid this problem, the $R$-parity violating parameters are constrained to be,

$$
\lambda, \lambda^{\prime}, \lambda^{\prime \prime}, \mu^{\prime} / \mu<\mathcal{O}\left(10^{-(6-7)}\right),
$$

where $\mu$ denotes the $R$-parity conserving $\mu$-parameter in the TeV range [33-36]. ${ }^{4}$ Hereafter, we suppress the family indices for simplicity.

\footnotetext{
${ }^{2}$ See [21] for a recent discussion on $R$-parity violation in string theory.

${ }^{3}$ This does not preclude the $R$-parity originating from symmetries other than $\mathrm{U}(1)_{B-L}$ though.

${ }^{4}$ See ref. [37], for baryogengesis [38, 39] in the presence of the $R$-parity violation.
} 
The bilinear $R$-parity terms in eq. (2.1) are also constrained from the neutrino mass [32]. By taking the cosmological upper limit on the neutrino mass, $\sum_{i} m_{\nu_{i}} \lesssim 0.183 \mathrm{eV}$ (at $95 \% \mathrm{CL}$ ) [40], the constraint is given by,

$$
\sum_{i} \frac{\mu^{\prime 2}}{\mu^{2}} \lesssim 2 \times 10^{-11} \tan ^{2} \beta\left(\frac{M_{2}}{1 \mathrm{TeV}}\right)\left(\frac{M_{1}}{M_{1} c_{W}^{2}+M_{2} s_{W}^{2}}\right) .
$$

Here, $M_{1,2}$ are the soft supersymmetry breaking masses of the bino and the wino, respectively, $\tan \beta$ is the ratio between the VEVs of the two Higgs doublets, and $s_{W}^{2}$ denotes the weak mixing angle, $\sin ^{2} \theta_{W}$, with $c_{W}^{2}=1-s_{W}^{2}$. It should be noted that we take the basis of the Higgs bosons and the sleptons, $\left(H_{d}, \tilde{L}_{i}\right)$, so that no sleptons obtain VEVs (see [32] for details).

Now, let us briefly discuss $R$-parity violation tied to $\mathrm{U}(1)_{B-L}$ breaking. In particular, we focus on models where the effects of $R$-parity violation in the MSSM appear through tiny VEVs of the right-handed sneutrinos, $\left\langle\tilde{N}_{R}\right\rangle[23,41]$ (see also the appendix A). In this class of models, the $R$-parity violating parameters are generated $\operatorname{as}^{5}$

$$
\mu^{\prime} \sim y_{\nu}\left\langle\tilde{N}_{R}\right\rangle
$$

Thus, the constraints in eqs. (2.2) and (2.3) on $\lambda^{(\prime)}$ and $\mu^{\prime}$ can be satisfied as long as $\left\langle N_{R}\right\rangle^{\prime} \mathrm{s}$ are small.

As an advantageous feature of this class of models, the $B$-violating term, $\lambda^{\prime \prime}$, can be further suppressed by additional symmetries (see the appendix A). Thus, this class of models can evade the sever constraints from the null observation of proton decay [32],

$$
\left|\lambda^{\prime} \lambda^{\prime \prime}\right| \lesssim 10^{-25}\left(\frac{m_{\mathrm{SUSY}}}{1 \mathrm{TeV}}\right)^{2}
$$

where $m_{\text {SUSY }}$ denotes a typical mass of superparticles.

When the $R$-parity violation effects are dominated by the bilinear terms, the gravitino mainly decays into a pair of a $Z$ boson and a neutrino, a pair of a Higgs boson and a neutrino, and a pair of a $W$ boson and a charged lepton. The relative branching ratios into those modes converge to $1: 1: 2$ in the limit of $m_{3 / 2} \gg m_{Z, W, h}[42-45]$. The decay widths of those modes are roughly given by,

$$
2 \Gamma\left[\psi_{3 / 2} \rightarrow Z \nu\right] \sim 2 \Gamma\left[\psi_{3 / 2} \rightarrow h \nu\right] \sim \Gamma\left[\psi_{3 / 2} \rightarrow W \ell\right] \sim \frac{m_{3 / 2}^{3}}{192 \pi M_{\mathrm{PL}}^{2}}\left(\frac{\mu^{\prime}}{\mu}\right)^{2},
$$

leading to the lifetime of the gravitino,

$$
\tau_{3 / 2} \simeq 10^{20} \sec \times\left(\frac{1 \mathrm{TeV}}{m_{3 / 2}}\right)^{3}\left(\frac{10^{-7} \mu}{\mu^{\prime}}\right)^{2} .
$$

Here, $m_{3 / 2}$ denotes the gravitino mass, and $M_{\mathrm{PL}} \simeq 2.4 \times 10^{18} \mathrm{GeV}$ the reduced Planck scale. Therefore, the lifetime of the gravitino can be much longer than the age of the universe, $\mathcal{O}\left(10^{17}\right)$ sec, for $\mu_{i}^{\prime} / \mu \ll 10^{-7}$ for the graivitino in the hundreds $\mathrm{GeV}$ to a TeV range.

\footnotetext{
${ }^{5}$ In the basis of $\left(H_{d}, \tilde{L}_{i}\right)$ where no sleptons obtain VEVs, the trilinear terms are also generated as $\lambda \sim \lambda^{\prime} \sim \mu^{\prime} / \mu$.
} 
The gravitino lifetime in the range of eq. (2.7) is, however, severely constrained from the observation of the extragalactic gamma-ray background (EGRB) [46-50]. ${ }^{6}$ By using 50-month EGRB observation by Fermi-LAT [52], the lifetime of the gravitino dark matter decaying into a pair of a $W$ boson and a charged lepton is constrained to be $\tau_{3 / 2} \gtrsim 10^{28}$ sec for $m_{3 / 2}=\mathcal{O}(100 \mathrm{GeV}-1 \mathrm{TeV})$. In the following, we assume that the bilinear $R$-parity violating parameters satisfy

$$
\frac{\mu^{\prime}}{\mu} \lesssim 10^{-11} \times\left(\frac{1 \mathrm{TeV}}{m_{3 / 2}}\right)^{3 / 2}
$$

\section{3 gravitino dark matter in thermal leptogensis scenario}

\subsection{Thermal gravitino production}

The productions of the gravitino from the thermal bath are dominated by the QCD process. The resultant relic density of the gravitino dark matter is given by [53, 54],

$$
\begin{aligned}
\Omega_{3 / 2} h^{2} \simeq 0.09\left(\frac{m_{3 / 2}}{100 \mathrm{GeV}}\right)\left(\frac{T_{R}}{10^{10} \mathrm{GeV}}\right) & \left(\left(1+0.558 \frac{r_{\tilde{g}}^{-2} m_{\tilde{g}}^{2}}{m_{3 / 2}^{2}}\right)\right. \\
& \left.-0.011\left(1+3.062 \frac{r_{\tilde{g}}^{-2} m_{\tilde{g}}^{2}}{m_{3 / 2}^{2}}\right) \log \left[\frac{T_{R}}{10^{10} \mathrm{GeV}}\right]\right),
\end{aligned}
$$

for the universal gaugino mass generated at the GUT scale. ${ }^{7}$ Here, the parameter $r_{\tilde{g}}$ is introduced to translate the universal gaugino mass parameter, $m_{1 / 2}$, at the GUT scale to the physical gluino mass, $m_{\tilde{g}}$,

$$
m_{\tilde{g}}=r_{\tilde{g}} m_{1 / 2},
$$

which depends on the MSSM parameters. In figure 1, we show $r_{\tilde{g}}$ as a function of the physical gluino mass calculated with the code SOFTSUSY [56] where the upper and lower bound correspond to squark mass $10 \mathrm{TeV}$ and $1 \mathrm{TeV}$. Here, we fix $\beta=10$ although $r_{\tilde{g}}$ barely depends on $\tan \beta$ nor other model parameters than $m_{1 / 2}$ and the squark masses. In our analysis, we adopt the upper bound of the figure 1 for a given $m_{\tilde{g}}$, which makes the following analysis conservative. It should be noted that the relic density in eq. (3.1) is about a factor two larger than the one in [30] which is caused by the thermal mass effects of the gluon as discussed in [53].

From the relic density in eq. (3.1), we immediately find that the gluino mass is severely constrained from above for successful leptogenesis which requires a high reheating temperature, $T_{R} \gtrsim 1.4 \times 10^{9} \mathrm{GeV}[57] .^{8}$ Here, we assume that the spectrum of the right-handed neutrinos are not degenerated. In figure 2 , we show the upper limits on the gluino mass for

\footnotetext{
${ }^{6}$ The observations of the neutrino fluxes also constraints the lifetime of the gravitino, which is less stringent than those from the EGRB [51].

${ }^{7}$ Electroweak contributions to the gravitino production increases the abundance by around $20 \%$ when the gaugino masses satisfy the GUT relation [55].

${ }^{8}$ The definitions of the reheating temperature in $[27,57]$ and in [54] are slightly different and the former is about $30 \%$ larger for a given inflaton decay width.
} 


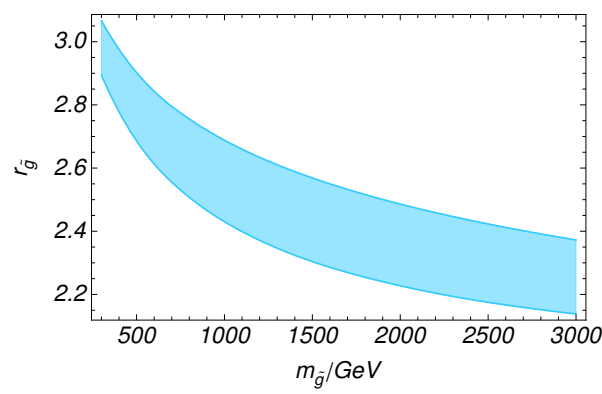

Figure 1. The parameter $r_{\tilde{g}}$ as a function of the physical gluino mass, $m_{\tilde{g}}$. The upper and lower bound correspond to $10 \mathrm{TeV}$ and $1 \mathrm{TeV}$ squark mass. We fix $\beta=10$ although $r_{\tilde{g}}$ barely depends on $\tan \beta$ nor other model parameters than $m_{1 / 2}$ and the squark masses.
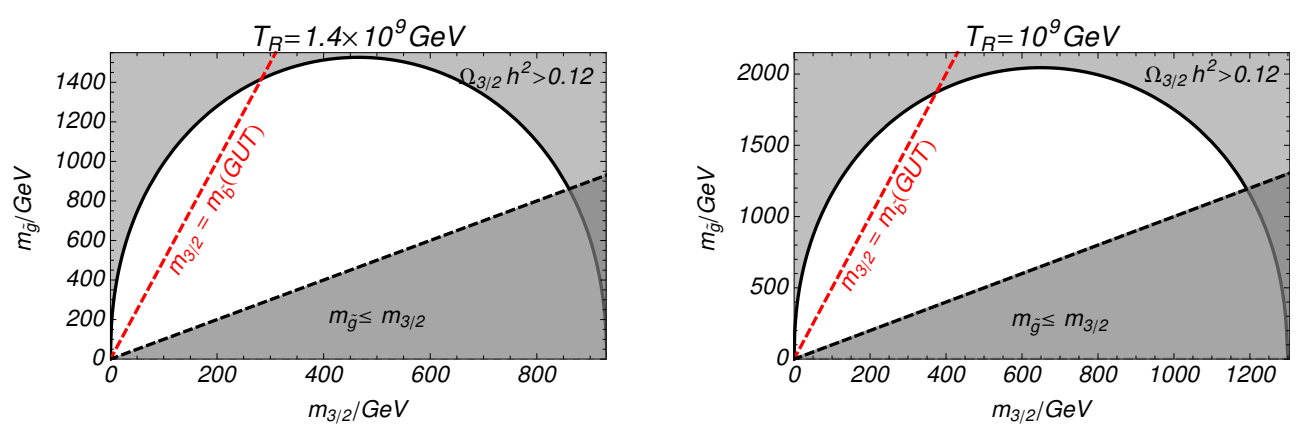

Figure 2. The upper limits on the gluino mass as a function of the gravitino mass, $m_{3 / 2}$, for given reheating temperatures. Below the black dashed lines, the gravitino becomes heavier than the gluino. The gray shaded regions are excluded where the gravitino abundance exceeds the observed dark matter abundance. The gaugino masses can satisfy the GUT relation in the left side of the red dashed line while keeping the gravitino being the LSP.

given reheating temperatures. In the figure, the gray shaded regions are excluded where the gravitino relic density exceeds the observed dark matter density, $\Omega h^{2} \simeq 0.1198 \pm 0.0015$ [58]. The dark matter density can be fully explained on the upper limit on the gluino mass for a given gravitino mass. The figure shows that the upper limit on the gluino mass is around $1.5 \mathrm{TeV}$ for $T_{R} \gtrsim 1.4 \times 10^{9} \mathrm{GeV}$. In the right panel of figure 2 , we also show the upper limit on the gluino mass for $T_{R} \simeq 10^{9} \mathrm{GeV}$ in case that the reheating temperature required for leptogenesis is somewhat relaxed.

Let us comment here that the constraints become much severer when the gaugino masses satisfy the GUT relation,

$$
m_{\tilde{b}}: m_{\tilde{w}}: m_{\tilde{g}} \sim 1: 2: 5-6,
$$

where $m_{\tilde{b}, \tilde{w}}$ are the masses of the bino and the wino, respectively. When the GUT relation is satisfied, the gravitino mass should be smaller than the bino mass

$$
m_{3 / 2}<m_{\tilde{b}} \simeq \frac{1}{5} m_{\tilde{g}},
$$


so that the gravitino is the LSP. In figure 2, this condition can be satisfied only in the left side of the red dashed line. There, the limits on the gluino mass are $m_{\tilde{g}} \lesssim 1.3 \mathrm{TeV}$ for $T_{R}>1.4 \times 10^{9} \mathrm{GeV}$ and $m_{\tilde{g}} \lesssim 1.8 \mathrm{TeV}$ for $T_{R}>10^{9} \mathrm{GeV}$, respectively.

In the right side of the red dashed line, on the other hand, the bino and the wino should be heavier than the GUT relation in order for the gravitino to be the LSP. Since the heavier bino/wino masses increase the electroweak contributions to the gravitino abundance, the abundance in eq. (3.1) underestimates the gravitino abundance in this region. Therefore, the upper limit on the gluino mass in the right side of the red dashed line is rather conservative.

\subsection{NLSP contributions}

As discussed in [59-63], the late-time decay of the next-to-lightest superparticle (NLSP) also contributes to the relic gravitino density,

$$
\Omega_{3 / 2}^{\text {tot }} h^{2}=\Omega_{3 / 2} h^{2}+B r_{3 / 2} \times \frac{m_{3 / 2}}{m_{\mathrm{NLSP}}} \Omega_{\mathrm{NLSP}} h^{2} .
$$

Here, $\Omega_{\mathrm{NLSP}}$ denotes the thermal relic density of the NLSP when it is stable, and $B r_{3 / 2}$ the branching fraction of the NLSP into the gravitino. In the absence of $R$-parity violation, the NLSP dominantly decays into the gravitino, and hence, $B r_{3 / 2}=1$. In this case, the constraints on the gluino mass from the gravitino abundance in figure 2 are severer [61, 64, 65].

In the presence of $R$-parity violation, on the contrary, $B r_{3 / 2}$ can be much suppressed when the effects of $R$-parity violation are sizable. In fact, the width of the $R$-parity violating decay (via the bilinear $R$-parity violating terms) is roughly given by,

$$
\Gamma_{\mathrm{NLSP}}^{R} \simeq \frac{\kappa}{16 \pi}\left(\frac{\mu^{\prime}}{\mu}\right)^{2} m_{\mathrm{NLSP}}
$$

which leads to the lifetime,

$$
\tau_{\mathrm{NLSP}}^{\not{K}} \simeq 10^{-4} \mathrm{sec} \times \kappa^{-1}\left(\frac{10^{-11} \mu}{\mu^{\prime}}\right)^{2}\left(\frac{1 \mathrm{TeV}}{m_{\mathrm{NLSP}}}\right) .
$$

Here, $\kappa$ represents dependences on the MSSM parameters [22, 42]. This width is much larger than the width of the $R$-parity conserving decay into a pair of the gravitino and the superpartner of the NLSP,

$$
\Gamma_{\mathrm{NLSP}}^{R} \simeq \frac{1}{48 \pi} \frac{m_{\mathrm{NLSP}}^{5}}{m_{3 / 2}^{2} M_{P L}^{2}},
$$

which corresponds to

$$
\tau_{\mathrm{NLSP}}^{R} \simeq 5 \times 10^{3} \sec \left(\frac{m_{3 / 2}}{100 \mathrm{GeV}}\right)^{2}\left(\frac{1 \mathrm{TeV}}{m_{\mathrm{NLSP}}}\right)^{5} .
$$

Therefore, $B r_{3 / 2}$ is expected to be very small even for small $R$-parity violating bilinear terms as in eq. (3.10).

It should be also noted that the properties of the NLSP are strongly constrained by Big-Bang nucleosynthesis (BBN) [66, 67]. For the neutralino NLSP, for example, the 
lifetime should be shorter than $10^{2}$ sec to avoid dissociation of the light elements by the NLSP decays into hadronic showers (especially into nucleons). For the stau NLSP, on the other hand, the lifetime should be shorter than $10^{3-4}$ sec to avoid the light element dissociation. By taking those constraints from the BBN into account, we assume that the $R$-parity violating parameters are in the range of,

$$
10^{-14} \times \kappa^{-1 / 2}\left(\frac{1 \mathrm{TeV}}{m_{\mathrm{NLSP}}}\right)^{1 / 2} \lesssim \frac{\mu^{\prime}}{\mu} \lesssim 10^{-11} \times\left(\frac{1 \mathrm{TeV}}{m_{3 / 2}}\right)^{3 / 2}
$$

In the appendix $\mathrm{A}$, we show a model which leads to the bilinear $R$-parity violation terms in this range. It should be noted that $B r_{3 / 2} \ll 1$ in this range of $R$-parity violation, and hence, the NLSP contribution to the gravitino abundance in eq. (3.5) is negligible. ${ }^{9}$

\subsection{Collider constraints}

In this subsection, we discuss the constraints from the superparticle searches at the LHC. First, let us note that the $R$-parity violating parameters we are interested in are small and we can apply the search strategies for the superparticles at the LHC in the $R$-conserving case. ${ }^{10}$ In fact, for the neutralino NLSP, the lifetime is typically given by [22, 42],

$$
c \tau_{\tilde{\chi}_{1}^{0}} \gtrsim 10^{6} \mathrm{~m} \times\left(\frac{1 \mathrm{TeV}}{m_{\tilde{\chi}_{1}^{0}}}\right)^{3}\left(\frac{10^{-11} \mu}{\mu^{\prime}}\right)^{2}\left(\frac{10}{\tan \beta}\right)^{2} .
$$

For the stau NLSP, on the other hand, the lifetime is similarly given by [22, 42],

$$
c \tau_{\tilde{\tau}} \gtrsim 10^{7} \mathrm{~m} \times\left(\frac{1 \mathrm{TeV}}{m_{\tilde{\tau}}}\right)\left(\frac{10^{-11} \mu}{\mu^{\prime}}\right)^{2}\left(\frac{10}{\tan \beta}\right)^{2} .
$$

Therefore, the NLSP is stable inside the detectors in both cases.

Let us begin with the collider constraints in the case of the neutralino NLSP. Since the neutralino NLSP is stable inside the detectors, we consider the searches for multi-jets with missing momentum. To derive conservative limits on the gluino mass, we assume that all the squarks are heavy and decoupled. In figure 3, we show the constraints on the gluino mass and the neutralino mass at the 95\% CL which are extracted from the results by the ATLAS collaboration [69]. ${ }^{11}$ Here, the gluino is assumed to decay into two quarks and a neutralino for simplicity. The figure shows that the lower limit on the gluino mass is around $1.8 \mathrm{TeV}$ when the neutralino is not degenerated with the gluino. When the neutralino mass is close to the gluino mass, the constraints become weaker though the the gluino mass below $1 \mathrm{TeV}$ is excluded unless the neutralino is highly degenerated with the gluino. It should be noted that the neutralino-gluino degenerated region is also excluded by the mono-jet searches up to $600 \mathrm{GeV}$ [70].

For the stau NLSP, on the other hand, we consider the long-lived charged particle searches. So far, the CMS collaboration puts a lower limit on the mass of the long-lived

\footnotetext{
${ }^{9}$ For typical size of $\Omega_{\mathrm{NLSP}} h^{2}$, see [61].

${ }^{10}$ See also [68] for the effects of $R$-parity violation on the LHC search for much lighter gravitinos.

${ }^{11}$ See also [73], for the constraints put by the CMS collaboration.
} 

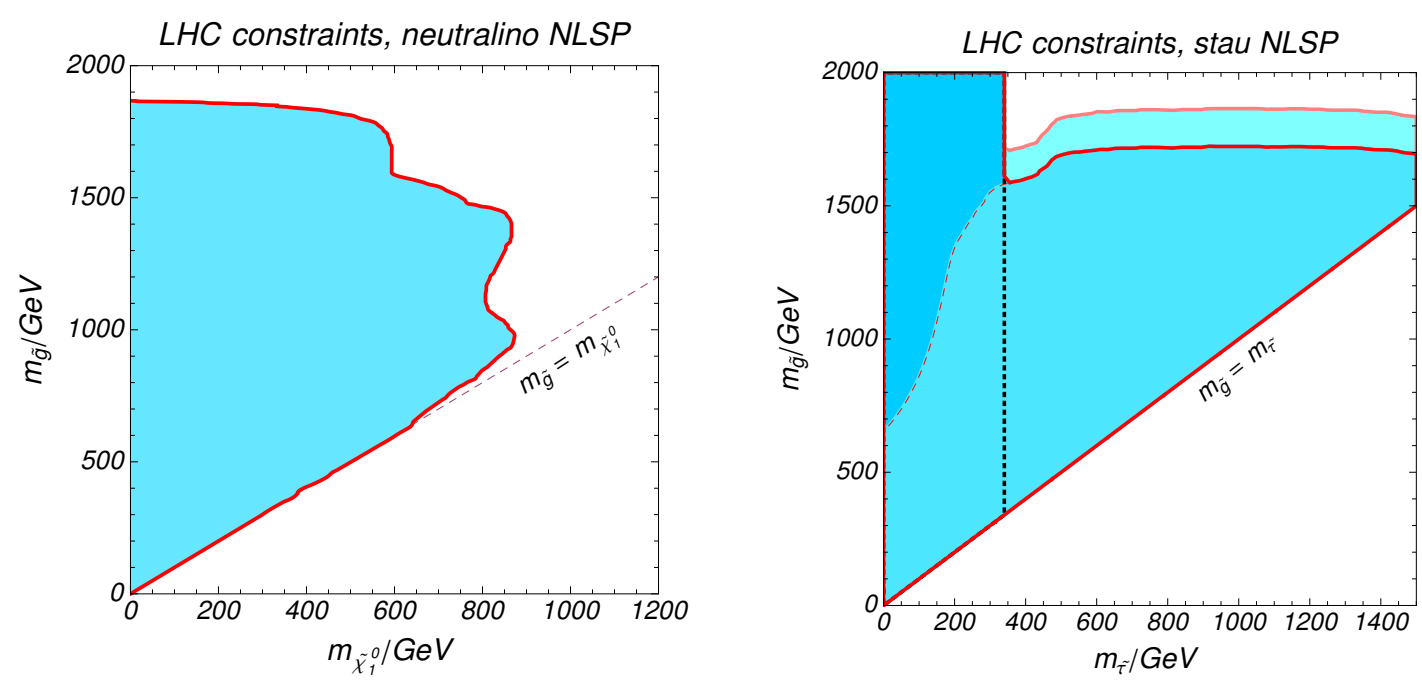

Figure 3. Left) The constraints on $\left(m_{\tilde{\chi}_{1}^{0}}, m_{\tilde{g}}\right)$ from the searches for multi-jets with missing momentum extracted from [69]. The neutralino-gluino degenerated region is also excluded by the mono-jet searches up to $600 \mathrm{GeV}$ [70]. Right) The constraints on $\left(m_{\tilde{\tau}}, m_{\tilde{g}}\right)$ from the searches for long-lived charged particles. The constraint on the stau production cross section in [71] is converted to the gluino mass bound by using the gluino NLO+NLL production cross section at $13 \mathrm{TeV}$ (reduced by $2 \sigma$ theoretical uncertainties) in [72]. The region with $m_{\tilde{\tau}}<340 \mathrm{GeV}$ is also excluded by the long-lived charged particle searches by assuming direct stau production [71]. It is noted that the region below $m_{\tilde{g}} \lesssim 1.5 \mathrm{TeV}$ with stable gluino where stau and gluino mass is (almost) degenerate is also excluded by R-hadron search [71]. In both panels, we assume that the squarks are heavy and decoupled. In both panels, we assume that constraints are obtained in the limit of decoupled squarks and therefore no dependence of the squark mass is present.

stau, $m_{\tilde{\tau}}>340 \mathrm{GeV}$ at $95 \% \mathrm{CL}$, by assuming a direct Drell-Yan stau pair production [71]. The CMS collaboration also puts upper limits on the production cross section of the stau pairs for a given stau mass. Since the stau production cross section (including the one from the cascade decays of the gluinos) depends on the gluino mass, we can put constraints on the gluino mass for a given stau mass. In figure 3, we show the resultant constraints on $\left(m_{\tilde{\tau}}, m_{\tilde{g}}\right)$ plane. Here, we obtain the constraints by comparing the $95 \%$ CL limits on the stau production cross section in [71] with the gluino production cross section in [72]. The light shaded region denotes the excluded region for the central value of the gluino NLO+NLL production cross section at $13 \mathrm{TeV}$ in [72], while the darker shaded region denotes the one for the cross section reduced by $2 \sigma$ theoretical uncertainties due to variation of the renormalization and factorization scales and the parton distribution functions. It is noted that the region below $m_{\tilde{g}} \lesssim 1.5 \mathrm{TeV}$ with stable gluino where stau and gluino mass is (almost) degenerate is also excluded by the $R$-hadron searches which we discuss more detail later in this section. In the following analysis, we use the later constraint for conservative estimation.

Now, let us combine the constraints from the gravitino abundance in figure 2 with the constraints in figure 3 from the collider searches. ${ }^{12}$ In figure 4 , we show the constraints

\footnotetext{
${ }^{12}$ In our analysis, we require that the dark matter density is dominated by the gravitino density. Hence,
} 

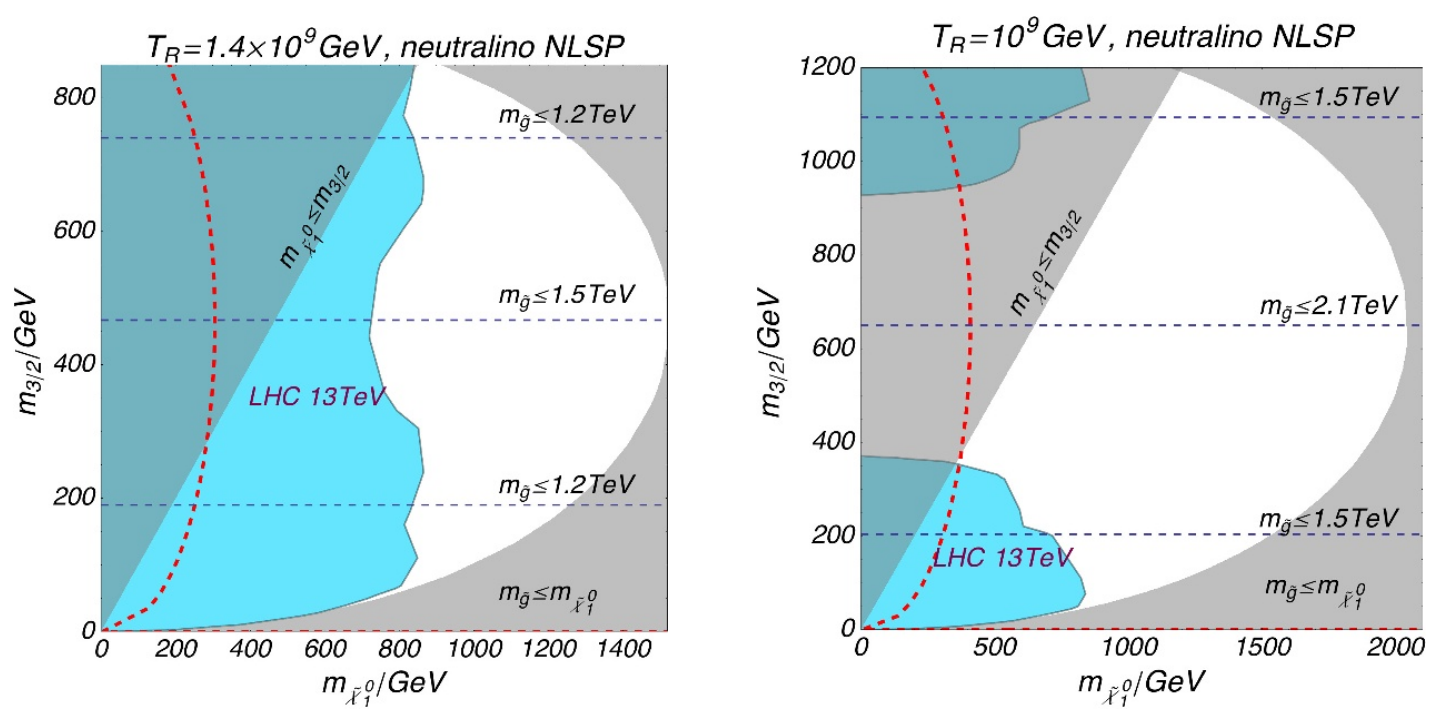

Figure 4. Combined constraints for the neutralino NLSP. The reheating temperature is assumed to be $T_{R}=1.4 \times 10^{9} \mathrm{GeV}$ (left) and $T_{R}=10^{9} \mathrm{GeV}$ (right). The gray shaded regions are excluded where the gravitino is no more the LSP. The blue shaded regions are excluded by the missing momentum searches $[69,73]$. The GUT relation of the gaugino mass can be satisfied in the left side of the red dashed line. The horizontal dashed lines show the upper limit on the gluino mass for a given gravitino mass shown in figure 2 .

in the case of the neutralino NLSP on the $\left(m_{\tilde{\chi}_{1}^{0}}, m_{3 / 2}\right)$ plane. The figure shows that large portion of the parameter region has been excluded by the LHC constraints for successful leptogenesis, i.e. $T_{R} \gtrsim 1.4 \times 10^{9} \mathrm{GeV}$. Even for somewhat relaxed requirement, $T_{R} \gtrsim$ $10^{9} \mathrm{GeV}$, some portion of the parameter region has been excluded by the LHC results. The remaining allowed region will be tested for $300 \mathrm{fb}^{-1}$ of integrated luminosity at $14 \mathrm{TeV}$ which reaches to $m_{\tilde{g}} \simeq 2.8 \mathrm{TeV}$ [74]. If we assume the GUT relation to the gaugino masses, the parameter region has been excluded even for somewhat lower reheating temperature $T_{R} \gtrsim 10^{9} \mathrm{GeV}$.

In figure 5, we also show the combined constraints for the stau NLSP. The figure shows that all the parameter region has been excluded by the LHC constraints for $T_{R} \gtrsim$ $1.4 \times 10^{9} \mathrm{GeV}$. For a relaxed requirement, $T_{R} \gtrsim 10^{9} \mathrm{GeV}$, on the other hand, there remains some allowed region, which can be also tested by further data taking.

So far, we have discussed the cases with the neutralino NLSP and the stau NLSP. Before closing this section, let us comment on other candidates for the NLSP. When the gluino is the NLSP, it is again stable inside the detectors, and is hadronized with the SM quarks to form the so-called $R$-hadrons $[75,76]$. The $R$-hadrons are charged unless the gluinos are bounded with the gluons, and the charged $R$-hadrons can be searched for as long-lived charged particles. So far, the CMS collaboration has excluded the gluino mass below $1.5 \mathrm{TeV}$ at $95 \% \mathrm{CL}$ when the $50 \%$ of the $R$-hadrons are assumed to be charged [71]. ${ }^{13}$ Thus, by comparing with the upper limit on the gluino mass in figure 2 , we find that the

we assume that the gluino mass should lie on the upper limit in figure 2 for a given gravitino mass.

${ }^{13}$ When the $90 \%$ of the $R$-hadrons are assumed to be charged, the constraints becomes $m_{\tilde{g}}<1.59 \mathrm{TeV}$. 

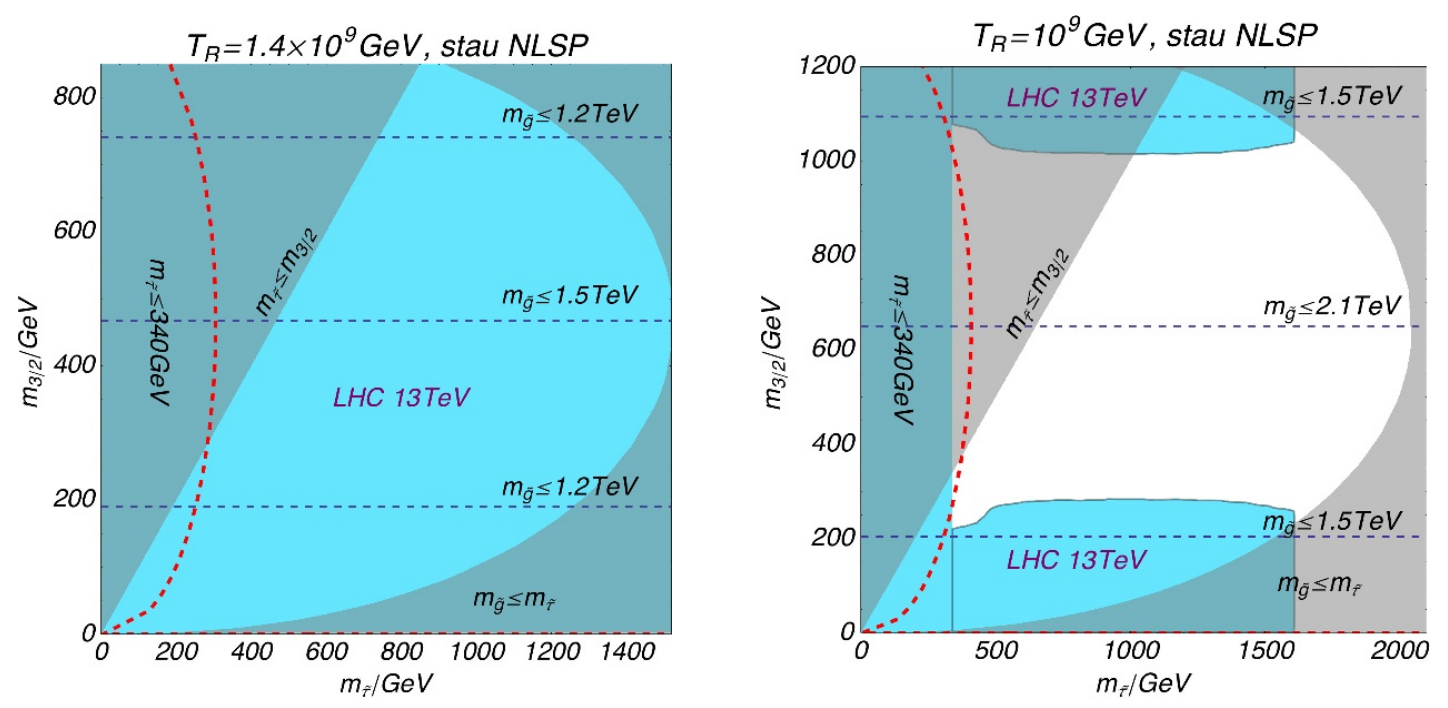

Figure 5. The same with 4 but for the stau NLSP.

gravitino dark matter cannot be consistent with the thermal leptogenesis as in the case with the stau NLSP, unless the required reheating temperature is somewhat lowered.

The CMS collaboration also puts constraints on the production cross section of the charged $R$-hadron assuming a stable stop particle [71], which can be applied to the cases of the stop NLSP and other squark NLSPs. Since the upper limits on the cross section for a given NLSP mass are tighter than the case of the gluino NLSP, the lower limits on the gluino mass are tighter for the squark/stop NLSP. Therefore, we again find that the gravitino dark matter cannot be consistent with the thermal leptogenesis for these NLSP candidates.

As for the other charged NLSPs such as selectron/smuon/charginos, the same constraints with the stau NLSP can be applied. For the sneutrino NLSP, on the other hand, it leaves missing momentum inside the detector as in the case of the neutralino NLSP. However, we need to perform more detailed analyses including model building to derive constraints, since the event topologies depend on the decay patterns of the gluinos into the sneutrinos. In addition, some careful parameter tunings are required to achieve the sneutrino NLSP in the MSSM. From these points of view, we do not pursue this possibility in this paper.

Finally, let us comment on models with a lighter gravitino. In our discussion, we have assumed that the gravitino is in the hundreds $\mathrm{GeV}$ to a few $\mathrm{TeV}$ range, where the NLSP decays outside of the detectors due to a limited size of $R$-parity violation as in eq. (2.8). If the gravitino mass is a few tens of $\mathrm{GeV}$, on the other hand, the bilinear $R$-parity violation terms can be as large as $\mu^{\prime} / \mu \sim 10^{-(7-8)} .{ }^{14}$ In such cases, the NLSP lifetime can be as short as $\mathcal{O}\left(10^{-9}\right)$ sec in the bi-linear $R$-parity violation [22]. Thus, the

\footnotetext{
${ }^{14}$ For such a light gravitino, it mainly decays into a pair of a photon and a neutrino. The lifetime of such a gravitino is constrained to be $\tau_{3 / 2} \gtrsim 10^{29} \mathrm{sec}$ by the searches for monochromatic gamma-ray line from the Galactic center region [77].
} 
neutral NLSP leaves a displaced vertex inside the detectors, and the charged NLSP leaves a kink inside the detectors. ${ }^{15}$ For such a light gravitino, however, the gravitino abundance requires $m_{\tilde{g}} \lesssim 500 \mathrm{GeV}$ which are severely constrained by the searches for multi-track displaced vertices for the neutral NLSP [81, 82] and by careful reinterpretation [83] of the disappearing track searches for the charged NLSP [84, 85]. We leave detailed analysis for such a light gravitino for future work.

\section{Conclusions and discussions}

In this paper, we revisited the gravitino dark matter scenario in the presence of the bilinear $R$-parity violating interactions. In particular, we discussed the consistency with the thermal leptogenesis. For a high reheating temperature required for the thermal leptogenesis, the gravitino dark matter tends to be overproduced, which puts a severe upper limit on the gluino mass. As a result, we found that a large portion of parameter space has been excluded by the null results of the searches for multi-jets with missing momentum at the LHC experiments when the NLSP is assumed to be the neutralino. For the stau (and other charged slepton) NLSP, on the other hand, more stringent constraints are put by the searches for the long-lived charged particles at the LHC experiments. As a result, almost all the parameter space has been excluded unless the required reheating temperature is somewhat lowered by assuming, for example, a degenerated right-handed neutrino spectrum. For the colored NLSP candidates, constraints are tighter than the ones for the stau NLSP, and hence, the gravitino dark matter cannot be consistent with thermal leptogenesis in those cases, neither.

It should be noted that the constraints from cosmology are more stringent in the absence of the $R$-parity violation since the late-time decay of the NLSP contributes to the gravitino dark matter abundance $[61,64,65]$. In addition, the properties of the NLSP are also constrained very severely by the BBN due to a long lifetime of the NLSP in the absence of $R$-parity violation. As a result, the successful BBN precludes the NLSP candidates other than the charged sleptons or the sneutrinos [61]. As for the charged sleptons, however, the parameter region has been excluded by the LHC results as discussed in this paper. The study of the sneutrino NLSP is a future work as mentioned above.

In our discussion, we focused on the bilinear $R$-parity violating interactions which are expected to be dominant in wide range of models of spontaneous $R$-parity breaking with the right-handed neutrinos. ${ }^{16}$ In fact, once $R$-parity is broken, the linear terms of the right-handed neutrinos, $\varepsilon_{R} N_{R}$, are generically allowed in the superpotential, which leads to $\langle N\rangle_{R} \sim \varepsilon_{\mathscr{R}} / M_{R}$. Here, $\varepsilon_{\not{R}}$ denotes an $R$-parity violating parameter and $M_{R}$ the right-handed neutrino mass. Therefore, the resultant bilinear $R$-parity violating terms are enhanced by $M_{R}^{-1}$ compared with trilinear $R$-parity violating terms which are suppressed not by $M_{R}$ but by higher cutoff scales such as the Planck scale or the GUT scale depending on the models.

\footnotetext{
${ }^{15}$ See e.g. [78-80] for discussions on the short lived NLSP's.

${ }^{16}$ See e.g. discussion in [86].
} 
Nontheless, when $R$-parity violation is dominated by trilinear terms, ${ }^{17}$ the gravitino decays into three SM fermions at a tree-level and into a pair of a SM boson and a fermion at the one loop level $[88,89]$. In those cases, the upper limits on the sizes of $R$-parity violation from EGRB can be weaker than that in eq. (2.8). Determination of the upper limits on $R$-parity violation requires more careful analyses in those cases. If the constraints can be relaxed to the ones in eq. (2.2), ${ }^{18}$ for example, the NLSP can decay promptly inside the detectors, which relax the LHC constraints. We leave such studies for future work.

\section{Acknowledgments}

T.T.Y. thanks Prof. Johannes Blumlein for hospitality during his stay at DESY in Zeuthen. This work is supported in part by Grants-in-Aid for Scientific Research from the Ministry of Education, Culture, Sports, Science, and Technology (MEXT) KAKENHI, Japan, No. 25105011 and No. 15 H05889 (M.I.) as well as No. 26104009 (T.T.Y.); Grant-in-Aid No. 26287039 (M.I. and T.T.Y.) and No. 16H02176 (T.T.Y.) from the Japan Society for the Promotion of Science (JSPS) KAKENHI; and by the World Premier International Research Center Initiative (WPI), MEXT, Japan (M.I., and T.T.Y.).

\section{A A model of $\boldsymbol{R}$-parity violation}

\section{A.1 $R$-parity violation tied with $\mathrm{U}(1)_{B-L}$ breaking}

In this appendix, we construct a model where the bilinear $R$-parity violating operators,

$$
W=\mu_{i}^{\prime} H_{u} L_{i}
$$

appear in the range of eq. (3.10) which are appropriate for the gravitino dark matter in the hundreds $\mathrm{GeV}$ to a $\mathrm{TeV}$ range. In particular, we consider a model where the $R$-parity violation is tied to a gauged $\mathrm{U}(1)_{B-L}$ breaking as motivated in $\mathrm{SO}(10)$ GUT models $[22,23]$.

Here, we use the $\mathrm{SO}(10)$ GUT notation, although it is straight forward to decompose the following discussion in terms of the MSSM fields. In the SO(10) GUT models, the quarks and leptons are grouped into $\mathbf{1 6}$ representation, $\mathbf{1 6}_{M}$, in conjunction with the right-handed neutrinos. The Higgs doublets are, on the other hand, grouped into $\mathbf{1 0}$ representation, $\mathbf{1 0}_{H}$. In our discussion, we do not specify the mechanisms which explain the doublet-triplet splitting of the Higgs multiplets, and we assume that only the Higgs doublets in $\mathbf{1 0}_{H}$ remain below the GUT scale.

In this notation, the MSSM Yukawa couplings are given by,

$$
W_{\mathrm{MSSM}}=\mathbf{1 0}_{H} \mathbf{1 6}_{M} 16_{M}
$$

where we have suppressed the coefficient and the family indices for simplicity. To give a large Majorana masses to the right-handed neutrinos, we need to introduce bilinear terms

\footnotetext{
${ }^{17}$ See e.g. a model in [87].

${ }^{18}$ Although this seems difficult unless the gravitino mass is below a few hundred GeV, which leads to a severer upper limits on the gluino mass as in figure 2 .
} 
of $\mathbf{1 6}_{M}$, which require at least a VEV of $\mathbf{1 2 6}$ representation. However, an introduction of a field in the $\mathbf{1 2 6}$ representation leads to a blow up of the $\mathrm{SO}(10)$ gauge coupling constant just above the GUT scale. To avoid this problem, we instead break the $\mathrm{U}(1)_{B-L} \subset \mathrm{SO}(10)$ by a VEV of $\overline{\mathbf{1 6}}_{H}$,

$$
\left\langle\overline{\mathbf{1 6}}_{H}\right\rangle=v_{B-L} .
$$

Here, as in the case of the Higgs doublets, we again assume that only the MSSM singlet in $\overline{\mathbf{1 6}}_{H}$. With the VEV of $\overline{\mathbf{1 6}}_{H}$, the Majorana mass terms are generated from,

$$
W_{\mathrm{N}_{\mathrm{R}}}=\frac{1}{2 M_{\mathrm{PL}}} \overline{\mathbf{1 6}}_{H} \overline{\mathbf{1 6}}_{H} \mathbf{1 6}_{M} \mathbf{1 6} \mathbf{6}_{M} .
$$

It should be noted no $\mathbb{Z}_{2}$ subgroup remains unbroken after $\mathrm{U}(1)_{B-L}$ breaking.

For a later purpose, we assume that $v_{B-L}=\mathcal{O}\left(10^{-(3-4)}\right) \times M_{\mathrm{PL}}$, so that the right handed neutrino masses are in the range of

$$
M_{R} \sim \frac{1}{M_{\mathrm{PL}}} v_{B-L}^{2} \sim 10^{-(6-8)} \times M_{\mathrm{PL}} .
$$

Then, we aim to construct a model where the right-handed redsneutrinos obtain VEVs

$$
\left\langle\tilde{N}_{R}\right\rangle \simeq \frac{v_{B-L}^{3}}{M_{\mathrm{PL}}^{3}} \times m_{3 / 2},
$$

while the $R$-parity conserving $\mu$-term is given by $\mu \sim m_{3 / 2}$. Once these are achieved, we obtain an appropriate bilinear $R$-parity violating terms,

$$
\frac{\mu^{\prime}}{\mu} \sim \frac{v_{B-L}^{3}}{M_{\mathrm{PL}}^{3}} .
$$

After the example of the model in [22], let us interconnect the $R$-parity violation to the $\mathrm{U}(1)_{B-L}$ breaking scale. First, in order to give a VEV to $\overline{\mathbf{1 6}}_{H}$, we consider a superpotential

$$
W=\mathbf{1 0}_{H} \mathbf{1 6}_{M} \mathbf{1 6}_{M}+\overline{\mathbf{1 6}}_{H} \overline{\mathbf{1 6}}_{H} \mathbf{1 6}_{M} \mathbf{1 6}_{M}+X\left(\mathbf{1 6}_{H} \overline{\mathbf{1 6}}_{H}-v_{B-L}^{2}\right),
$$

where $\mathbf{1 6} \mathbf{6}_{H}$ is a newly introduced $\mathbf{1 6}$ representation and $X$ is an $\mathrm{SO}(10)$ singlet. Hereafter, we take the unit of $M_{\mathrm{PL}}=1$. The first term of eq. (A.8) again denotes the MSSM Yukawa interaction, and the second term the Majorana mass term of the right-handed neutrinos. By the assumption of the sprit multiplet, $\mathbf{1 6}_{H}$ and $\overline{\mathbf{1 6}}_{H}$ contain the MSSM singlets only which are absorbed into $\mathrm{U}(1)_{B-L}$ gauge multiplet once they obtain the vacuum expectation value.

In order to avoid too large $R$-parity violations, we forbid the following operators

$$
\begin{aligned}
& W=\mathbf{1 6}_{M} \overline{\mathbf{1 6}}_{H}, \\
& W=\mathbf{1 0}_{H} \mathbf{1 6}_{M} \mathbf{1 6}_{H}, \\
& W=\mathbf{1 6}_{H} \mathbf{1 6}_{M} \mathbf{1 6}_{M} \mathbf{1 6} \mathbf{6}_{M} .
\end{aligned}
$$

For that purpose, we consider a continuos $R$-symmetry broken by a spurion $v_{B-L}$ (see more discussions in the next subsection). In table 1 , we show the $R$-charge assignment which forbids the above operators. 


\begin{tabular}{|c|ccccccc|}
\hline & $\mathbf{1 6}_{M}$ & $\mathbf{1 0}_{H}$ & $\mathbf{1 6}_{H}$ & $\overline{\mathbf{1 6}}_{H}$ & $v_{B-L}$ & $X$ & $m_{3 / 2}$ \\
\hline$R$ & 1 & 0 & $-1 / 2$ & 0 & $-1 / 4$ & $5 / 2$ & 2 \\
\hline
\end{tabular}

Table 1. $R$-charges of matter fields, Higgs fields and $\mathrm{SO}(10)$ singlets.

A notable feature of the $R$-charge assignment in table 1 is that it allows a Kähler potential,

$$
K=v_{B-L}^{4} \mathbf{1 6}_{M} \overline{\mathbf{1 6}}_{H},
$$

which leads to a linear term of the right-handed neutrinos ${ }^{19}$

$$
W \sim m_{3 / 2} v_{B-L}^{5} N_{R} .
$$

Thus, by combined with the Majorana mass term, the right-handed sneutrinos obtain VEVs,

$$
\left\langle\tilde{N}_{R}\right\rangle \simeq v_{B-L}^{3} \times m_{3 / 2}
$$

which generate the bilinear $R$-parity violation through the first term of eq. (A.8),

$$
\mu^{\prime}=v_{B-L}^{3} m_{3 / 2} .
$$

The $R$-symmetric $\mu$-term is, on the other hand, given by

$$
W \sim m_{3 / 2} \mathbf{1 0}_{H} \mathbf{1 0}_{H} .
$$

Therefore, we find that the bilinear $R$-parity violation are given by, ${ }^{20}$

$$
\frac{\mu^{\prime}}{\mu} \sim v_{B-L}^{3} .
$$

\section{A.2 Model with discrete $R$-symmetry}

In the above example, we made use of a continuous $R$-symmetry which is broken by the spurion $v_{B-L}$. In this subsection, we discuss a model where $v_{B-L}$ is dynamical. For that purpose, we consider $\mathrm{SU}(5)$ gauge theory with four-flavor of vector-like pairs of fundamental representation $(Q, \bar{Q})$, and replace $v_{B-L}^{2}$ to a composite operator $(Q \bar{Q})$. Then, the superpotential in eq. (A.8) is rewritten by,

$$
W=\mathbf{1 0}_{H} \mathbf{1 6}_{M} \mathbf{1 6}{ }_{M}+\overline{\mathbf{1 6}}_{H} \overline{\mathbf{1 6}}_{H} \mathbf{1 6}_{M} \mathbf{1 6}_{M}+X\left(\mathbf{1 6}_{H} \overline{\mathbf{1 6}}_{H}-Q \bar{Q}\right),
$$

where the $R$-charge assignment is given in table $2 .{ }^{21}$ Since the $\mathrm{SU}(5)$ gauge theory with four-flavor does not have a vacuum, we add explicit mass terms

$$
W=\mathbf{1 0}_{H} \mathbf{1 6}_{M} \mathbf{1 6}_{M}+\overline{\mathbf{1 6}}_{H} \overline{\mathbf{1 6}}_{H} \mathbf{1 6}_{M} \mathbf{1 6}_{M}+X\left(\mathbf{1 6}_{H} \overline{\mathbf{1 6}}_{H}-Q \bar{Q}\right)+m_{Q} Q \bar{Q} .
$$

With the explicit mass term, the $R$-symmetry is explicitly broken down to a discrete $\mathbb{Z}_{10 R}$ symmetry whose charge assignment is given in the second line of table $2 .{ }^{22}$

\footnotetext{
${ }^{19}$ If we regard $m_{3 / 2}$, we may directly write down this term.

${ }^{20}$ The actual $R$-parity violating bilinear terms are multiplied by the neutrino Yukawa couplings.

${ }^{21}$ This charge assignment is free from the $\mathrm{SU}(5)$ anomaly.

${ }^{22}$ As we will see, we require $m_{Q} \ll 1$. For that purpose, we assume that $m_{Q}, X, \mathbf{1 6}_{H}$ and $Q \bar{Q}$ are charged under some additional discrete symmetry.
} 


\begin{tabular}{|c|ccccccc|}
\hline & $\mathbf{1 6}_{M}$ & $\mathbf{1 0}_{H}$ & $\mathbf{1 6}_{H}$ & $\overline{\mathbf{1 6}}_{H}$ & $Q$ & $\bar{Q}$ & $X$ \\
\hline$R$ & 1 & 0 & $-1 / 2$ & 0 & $-1 / 4$ & $-1 / 4$ & $5 / 2$ \\
\hline $\mathbb{Z}_{10 R}$ & -4 & 0 & 2 & 0 & 1 & 1 & 0 \\
\hline
\end{tabular}

Table 2. $R$-charges of matter fields, Higgs fields and $\mathrm{SO}(10)$ singlets. We also show the charge assignment of $\mathbb{Z}_{10 R}$ with which the terms with charges $-8(\bmod 10)$ are allowed in the superpotential.

Below the dynamical scale of SU(5), non-perturbative potential is generated [90]

$$
\begin{aligned}
W= & \mathbf{1 0}_{H} \mathbf{1 6}_{M} \mathbf{1 6}_{M}+\overline{\mathbf{1 6}}_{H} \overline{\mathbf{1 6}}_{H} \mathbf{1 6}_{M} \mathbf{1 6}_{M}+X\left(\mathbf{1 6}_{H} \overline{\mathbf{1 6}}_{H}-Q \bar{Q}\right) \\
& +m_{Q} Q \bar{Q}+\frac{\Lambda^{11}}{\operatorname{det} Q \bar{Q}},
\end{aligned}
$$

where $\Lambda$ denotes the dynamical scale of $\mathrm{SU}(5)$. As a result, $Q \bar{Q}$ obtains a VEV, which provides the spurion in the previous section

$$
v_{B-L}^{2} \sim\langle Q \bar{Q}\rangle \sim\left(\frac{\Lambda^{11}}{m_{Q}}\right)^{1 / 5} .
$$

Thus, by arranging

$$
\left(\frac{\Lambda^{11}}{m_{Q}}\right)^{1 / 5}=\mathcal{O}\left(10^{-6}\right),
$$

we can provide an appropriate suprion $v_{B-L}=\mathcal{O}\left(10^{-3}\right)$. Furthermore, we can also provide an appropriate size of the gravition mass by taking

$$
m_{Q} \simeq 10^{-9}, \quad \Lambda \simeq 10^{-3.5},
$$

which leads to an appropriate VEV of the superpotential simultaneously

$$
m_{3 / 2}=\langle W\rangle \sim m_{Q}\langle Q \bar{Q}\rangle \simeq 10^{-15} .
$$

Open Access. This article is distributed under the terms of the Creative Commons Attribution License (CC-BY 4.0), which permits any use, distribution and reproduction in any medium, provided the original author(s) and source are credited.

\section{References}

[1] P. Fayet, Supersymmetry and Weak, Electromagnetic and Strong Interactions, Phys. Lett. B 64 (1976) 159 [INSPIRE].

[2] P. Fayet, Spontaneously Broken Supersymmetric Theories of Weak, Electromagnetic and Strong Interactions, Phys. Lett. B 69 (1977) 489 [INSPIRE].

[3] L.J. Hall and M. Suzuki, Explicit R-Parity Breaking in Supersymmetric Models, Nucl. Phys. B 231 (1984) 419 [INSPIRE].

[4] S.W. Hawking, Quantum Coherence Down the Wormhole, Phys. Lett. B 195 (1987) 337 [INSPIRE]. 
[5] G.V. Lavrelashvili, V.A. Rubakov and P.G. Tinyakov, Disruption of Quantum Coherence upon a Change in Spatial Topology in Quantum Gravity, JETP Lett. 46 (1987) 167 [INSPIRE].

[6] S.B. Giddings and A. Strominger, Loss of Incoherence and Determination of Coupling Constants in Quantum Gravity, Nucl. Phys. B 307 (1988) 854 [INSPIRE].

[7] S.R. Coleman, Why There Is Nothing Rather Than Something: A Theory of the Cosmological Constant, Nucl. Phys. B 310 (1988) 643 [InSPIRE].

[8] G. Gilbert, Wormhole induced proton decay, Nucl. Phys. B 328 (1989) 159 [InSPIRE].

[9] T. Banks and N. Seiberg, Symmetries and Strings in Field Theory and Gravity, Phys. Rev. D 83 (2011) 084019 [arXiv: 1011.5120] [InSPIRE].

[10] L.M. Krauss and F. Wilczek, Discrete Gauge Symmetry in Continuum Theories, Phys. Rev. Lett. 62 (1989) 1221 [INSPIRE].

[11] J. Preskill and L.M. Krauss, Local Discrete Symmetry and Quantum Mechanical Hair, Nucl. Phys. B 341 (1990) 50 [INSPIRE].

[12] J. Preskill, S.P. Trivedi, F. Wilczek and M.B. Wise, Cosmology and broken discrete symmetry, Nucl. Phys. B 363 (1991) 207 [InSPIRE].

[13] T. Banks and M. Dine, Note on discrete gauge anomalies, Phys. Rev. D 45 (1992) 1424 [hep-th/9109045] [INSPIRE].

[14] S. Dimopoulos and H. Georgi, Softly Broken Supersymmetry and SU(5), Nucl. Phys. B 193 (1981) 150 [INSPIRE].

[15] S. Weinberg, Supersymmetry at Ordinary Energies. 1. Masses and Conservation Laws, Phys. Rev. D 26 (1982) 287 [INSPIRE].

[16] N. Sakai and T. Yanagida, Proton Decay in a Class of Supersymmetric Grand Unified Models, Nucl. Phys. B 197 (1982) 533 [INSPIRE].

[17] S. Dimopoulos, S. Raby and F. Wilczek, Proton Decay in Supersymmetric Models, Phys. Lett. B 112 (1982) 133 [INSPIRE].

[18] T. Yanagida, Horizontal Symmetry And Masses Of Neutrinos, proceedings of Workshop on the Unified Theories and the Baryon Number in the Universe, Tsukuba, Japan, February 13-14, 1979 Conf. Proc. C7902131 (1979) 95 [INSPIRE].

[19] P. Ramond, The Family Group in Grand Unified Theories, hep-ph/9809459 [INSPIRE].

[20] P. Minkowski, $\mu \rightarrow$ e $\gamma$ at a Rate of One Out of $10^{9}$ Muon Decays?, Phys. Lett. B 67 (1977) 421 [INSPIRE].

[21] T. Watari, Statistics of F-theory flux vacua for particle physics, JHEP 11 (2015) 065 [arXiv: 1506.08433] [INSPIRE].

[22] W. Buchmüller, L. Covi, K. Hamaguchi, A. Ibarra and T. Yanagida, Gravitino Dark Matter in R-Parity Breaking Vacua, JHEP 03 (2007) 037 [hep-ph/0702184] [INSPIRE].

[23] J. Schmidt, C. Weniger and T.T. Yanagida, Dynamical Matter-Parity Breaking and Gravitino Dark Matter, Phys. Rev. D 82 (2010) 103517 [arXiv: 1008. 0398] [InSPIRE].

[24] F. Takayama and M. Yamaguchi, Gravitino dark matter without R-parity, Phys. Lett. B 485 (2000) 388 [hep-ph/0005214] [INSPIRE].

[25] G. Moreau and M. Chemtob, R-parity violation and the cosmological gravitino problem, Phys. Rev. D 65 (2002) 024033 [hep-ph/0107286] [INSPIRE]. 
[26] M. Fukugita and T. Yanagida, Baryogenesis Without Grand Unification, Phys. Lett. B 174 (1986) 45 [INSPIRE].

[27] G.F. Giudice, A. Notari, M. Raidal, A. Riotto and A. Strumia, Towards a complete theory of thermal leptogenesis in the SM and MSSM, Nucl. Phys. B 685 (2004) 89 [hep-ph/0310123] [INSPIRE].

[28] W. Buchmüller, R.D. Peccei and T. Yanagida, Leptogenesis as the origin of matter, Ann. Rev. Nucl. Part. Sci. 55 (2005) 311 [hep-ph/0502169] [INSPIRE].

[29] S. Davidson, E. Nardi and Y. Nir, Leptogenesis, Phys. Rept. 466 (2008) 105 [arXiv:0802.2962] [INSPIRE].

[30] M. Bolz, A. Brandenburg and W. Buchmüller, Thermal production of gravitinos, Nucl. Phys. B 606 (2001) 518 [Erratum ibid. B 790 (2008) 336] [hep-ph/0012052] [INSPIRE].

[31] K. Hamaguchi, F. Takahashi and T.T. Yanagida, Decaying gravitino dark matter and an upper bound on the gluino mass, Phys. Lett. B 677 (2009) 59 [arXiv:0901.2168] [INSPIRE].

[32] R. Barbier et al., R-parity violating supersymmetry, Phys. Rept. 420 (2005) 1 [hep-ph/0406039] [INSPIRE].

[33] T. Christodoulakis and E. Korfiatis, Contact transformations and the quantization of constraint systems, Phys. Lett. B 256 (1991) 457 [INSPIRE].

[34] W. Fischler, G.F. Giudice, R.G. Leigh and S. Paban, Constraints on the baryogenesis scale from neutrino masses, Phys. Lett. B 258 (1991) 45 [INSPIRE].

[35] H.K. Dreiner and G.G. Ross, Sphaleron erasure of primordial baryogenesis, Nucl. Phys. B 410 (1993) 188 [hep-ph/9207221] [INSPIRE].

[36] M. Endo, K. Hamaguchi and S. Iwamoto, Lepton Flavor Violation and Cosmological Constraints on R-parity Violation, JCAP 02 (2010) 032 [arXiv:0912.0585] [INSPIRE].

[37] T. Higaki, K. Nakayama, K. Saikawa, T. Takahashi and M. Yamaguchi, Affleck-Dine baryogenesis with R-parity violation, Phys. Rev. D 90 (2014) 045001 [arXiv:1404.5796] [INSPIRE].

[38] I. Affleck and M. Dine, A New Mechanism for Baryogenesis, Nucl. Phys. B 249 (1985) 361 [INSPIRE].

[39] M. Dine, L. Randall and S.D. Thomas, Baryogenesis from flat directions of the supersymmetric standard model, Nucl. Phys. B 458 (1996) 291 [hep-ph/9507453] [INSPIRE].

[40] E. Giusarma, M. Gerbino, O. Mena, S. Vagnozzi, S. Ho and K. Freese, Improvement of cosmological neutrino mass bounds, Phys. Rev. D 94 (2016) 083522 [arXiv:1605.04320] [INSPIRE].

[41] M. Ibe, S. Iwamoto, S. Matsumoto, T. Moroi and N. Yokozaki, Recent Result of the AMS-02 Experiment and Decaying Gravitino Dark Matter in Gauge Mediation, JHEP 08 (2013) 029 [arXiv: 1304.1483] [INSPIRE].

[42] K. Ishiwata, S. Matsumoto and T. Moroi, High Energy Cosmic Rays from the Decay of Gravitino Dark Matter, Phys. Rev. D 78 (2008) 063505 [arXiv:0805.1133] [InSPIRE].

[43] K. Ishiwata, S. Matsumoto and T. Moroi, Cosmic-Ray Positron from Superparticle Dark Matter and the PAMELA Anomaly, Phys. Lett. B 675 (2009) 446 [arXiv:0811.0250] [INSPIRE]. 
[44] K. Ishiwata, S. Matsumoto and T. Moroi, High Energy Cosmic Rays from Decaying Supersymmetric Dark Matter, JHEP 05 (2009) 110 [arXiv:0903.0242] [INSPIRE].

[45] T. Delahaye and M. Grefe, Antiproton limits on decaying gravitino dark matter, JCAP 12 (2013) 045 [arXiv: 1305.7183] [INSPIRE].

[46] A. Ibarra and D. Tran, Gamma Ray Spectrum from Gravitino Dark Matter Decay, Phys. Rev. Lett. 100 (2008) 061301 [arXiv: 0709.4593] [inSPIRE].

[47] K. Ishiwata, S. Matsumoto and T. Moroi, Cosmic Gamma-ray from Inverse Compton Process in Unstable Dark Matter Scenario, Phys. Lett. B 679 (2009) 1 [arXiv:0905.4593] [InSPIRE].

[48] E. Carquin, M.A. Diaz, G.A. Gomez-Vargas, B. Panes and N. Viaux, Confronting recent AMS-02 positron fraction and Fermi-LAT extragalactic $\gamma$-ray background measurements with gravitino dark matter, Phys. Dark Univ. 11 (2016) 1 [arXiv:1501.05932] [INSPIRE].

[49] S. Ando and K. Ishiwata, Constraints on decaying dark matter from the extragalactic gamma-ray background, JCAP 05 (2015) 024 [arXiv: 1502.02007] [INSPIRE].

[50] S. Ando and K. Ishiwata, Constraining particle dark matter using local galaxy distribution, JCAP 06 (2016) 045 [arXiv: 1604.02263] [INSPIRE].

[51] L. Covi, M. Grefe, A. Ibarra and D. Tran, Unstable Gravitino Dark Matter and Neutrino Flux, JCAP 01 (2009) 029 [arXiv: 0809.5030] [INSPIRE].

[52] Fermi-LAT collaboration, M. Ackermann et al., The spectrum of isotropic diffuse gamma-ray emission between $100 \mathrm{MeV}$ and 820 GeV, Astrophys. J. 799 (2015) 86 [arXiv: 1410.3696] [INSPIRE].

[53] V.S. Rychkov and A. Strumia, Thermal production of gravitinos, Phys. Rev. D 75 (2007) 075011 [hep-ph/0701104] [INSPIRE].

[54] J. Ellis, M.A.G. Garcia, D.V. Nanopoulos, K.A. Olive and M. Peloso, Post-Inflationary Gravitino Production Revisited, JCAP 03 (2016) 008 [arXiv:1512.05701] [INSPIRE].

[55] J. Pradler and F.D. Steffen, Thermal gravitino production and collider tests of leptogenesis, Phys. Rev. D 75 (2007) 023509 [hep-ph/0608344] [INSPIRE].

[56] B.C. Allanach, SOFTSUSY: a program for calculating supersymmetric spectra, Comput. Phys. Commun. 143 (2002) 305 [hep-ph/0104145] [INSPIRE].

[57] S. Antusch and A.M. Teixeira, Towards constraints on the SUSY seesaw from flavour-dependent leptogenesis, JCAP 02 (2007) 024 [hep-ph/0611232] [INSPIRE].

[58] Planck collaboration, P.A.R. Ade et al., Planck 2015 results. XX. Constraints on inflation, Astron. Astrophys. 594 (2016) A20 [arXiv: 1502.02114] [INSPIRE].

[59] J.L. Feng, A. Rajaraman and F. Takayama, Superweakly interacting massive particles, Phys. Rev. Lett. 91 (2003) 011302 [hep-ph/0302215] [INSPIRE].

[60] J.L. Feng, A. Rajaraman and F. Takayama, SuperWIMP dark matter signals from the early universe, Phys. Rev. D 68 (2003) 063504 [hep-ph/0306024] [INSPIRE].

[61] M. Fujii, M. Ibe and T. Yanagida, Upper bound on gluino mass from thermal leptogenesis, Phys. Lett. B 579 (2004) 6 [hep-ph/0310142] [INSPIRE].

[62] J.L. Feng, S.-f. Su and F. Takayama, SuperWIMP gravitino dark matter from slepton and sneutrino decays, Phys. Rev. D 70 (2004) 063514 [hep-ph/0404198] [INSPIRE]. 
[63] J.L. Feng, S. Su and F. Takayama, Supergravity with a gravitino LSP, Phys. Rev. D 70 (2004) 075019 [hep-ph/0404231] [INSPIRE].

[64] J. Heisig, Gravitino LSP and leptogenesis after the first LHC results, JCAP 04 (2014) 023 [arXiv: 1310.6352] [INSPIRE].

[65] A. Arbey, M. Battaglia, L. Covi, J. Hasenkamp and F. Mahmoudi, LHC constraints on Gravitino Dark Matter, Phys. Rev. D 92 (2015) 115008 [arXiv:1505.04595] [INSPIRE].

[66] M. Kawasaki, K. Kohri and T. Moroi, Big-Bang nucleosynthesis and hadronic decay of long-lived massive particles, Phys. Rev. D 71 (2005) 083502 [astro-ph/0408426] [INSPIRE].

[67] K. Jedamzik, Big bang nucleosynthesis constraints on hadronically and electromagnetically decaying relic neutral particles, Phys. Rev. D 74 (2006) 103509 [hep-ph/0604251] [INSPIRE].

[68] M. Hirsch, W. Porod and D. Restrepo, Collider signals of gravitino dark matter in bilinearly broken R-parity, JHEP 03 (2005) 062 [hep-ph/0503059] [INSPIRE].

[69] ATLAS collaboration, Further searches for squarks and gluinos in final states with jets and missing transverse momentum at $\sqrt{s}=13 \mathrm{TeV}$ with the ATLAS detector, ATLAS-CONF-2016-078 (2016).

[70] A. Arbey, M. Battaglia and F. Mahmoudi, Monojet Searches for MSSM Simplified Models, Phys. Rev. D 94 (2016) 055015 [arXiv: 1506.02148] [INSPIRE].

[71] CMS collaboration, Searchesrches for Long-lived Charged Particles in Proton-Proton Collisions at $\sqrt{s}=13 \mathrm{TeV}$, CMS-PAS-EXO-15-010 (2015).

[72] C. Borschensky et al., Squark and gluino production cross sections in pp collisions at $\sqrt{s}=13,14,33$ and $100 \mathrm{TeV}$, Eur. Phys. J. C 74 (2014) 3174 [arXiv:1407.5066] [inSPIRE].

[73] CMS collaboration, CMS-PAS-SUS-16-014 (2016).

[74] T. Cohen et al., A Comparison of Future Proton Colliders Using SUSY Simplified Models: A Snowmass Whitepaper, in proceedings of Community Summer Study 2013: Snowmass on the Mississippi (CSS2013) Minneapolis, MN, U.S.A., July 29 - August 6, 2013, arXiv: 1310.0077 [INSPIRE].

[75] G.R. Farrar, Status of light gaugino scenarios, Nucl. Phys. Proc. Suppl. 62 (1998) 485 [hep-ph/9710277] [INSPIRE].

[76] A.C. Kraan, J.B. Hansen and P. Nevski, Discovery potential of R-hadrons with the ATLAS detector, Eur. Phys. J. C 49 (2007) 623 [hep-ex/0511014] [InSPIRE].

[77] Fermi-LAT collaboration, M. Ackermann et al., Updated search for spectral lines from Galactic dark matter interactions with pass 8 data from the Fermi Large Area Telescope, Phys. Rev. D 91 (2015) 122002 [arXiv:1506.00013] [INSPIRE].

[78] S. Asai, Y. Azuma, M. Endo, K. Hamaguchi and S. Iwamoto, Stau Kinks at the LHC, JHEP 12 (2011) 041 [arXiv: 1103.1881] [INSPIRE].

[79] P.W. Graham, D.E. Kaplan, S. Rajendran and P. Saraswat, Displaced Supersymmetry, JHEP 07 (2012) 149 [arXiv: 1204.6038] [inSPIRE].

[80] C. Csáki, E. Kuflik, S. Lombardo, O. Slone and T. Volansky, Phenomenology of a Long-Lived LSP with R-Parity Violation, JHEP 08 (2015) 016 [arXiv: 1505.00784] [INSPIRE].

[81] ATLAS collaboration, Search for long-lived, heavy particles in final states with a muon and a multi-track displaced vertex in proton-proton collisions at $\sqrt{s}=8 \mathrm{TeV}$ with the ATLAS detector., ATLAS-CONF-2013-092 (2013). 
[82] ATLAS collaboration, Search for massive, long-lived particles using multitrack displaced vertices or displaced lepton pairs in pp collisions at $\sqrt{s}=8 \mathrm{TeV}$ with the ATLAS detector, Phys. Rev. D 92 (2015) 072004 [arXiv: 1504.05162] [INSPIRE].

[83] J.A. Evans and J. Shelton, Long-Lived Staus and Displaced Leptons at the LHC, JHEP 04 (2016) 056 [arXiv: 1601.01326] [InSPIRE].

[84] ATLAS collaboration, Search for charginos nearly mass degenerate with the lightest neutralino based on a disappearing-track signature in pp collisions at $\sqrt{s}=8 \mathrm{TeV}$ with the ATLAS detector, Phys. Rev. D 88 (2013) 112006 [arXiv:1310.3675] [INSPIRE].

[85] CMS collaboration, Search for disappearing tracks in proton-proton collisions at $\sqrt{s}=8 \mathrm{TeV}$, JHEP 01 (2015) 096 [arXiv: 1411.6006] [INSPIRE].

[86] S. Shirai, F. Takahashi and T.T. Yanagida, R-violating Decay of Wino Dark Matter and electron/positron Excesses in the PAMELA/Fermi Experiments, Phys. Lett. B 680 (2009) 485 [arXiv: 0905.0388] [INSPIRE].

[87] B. Bhattacherjee, J.L. Evans, M. Ibe, S. Matsumoto and T.T. Yanagida, Natural supersymmetry's last hope: R-parity violation via UDD operators, Phys. Rev. D 87 (2013) 115002 [arXiv:1301.2336] [INSPIRE].

[88] N.E. Bomark, S. Lola, P. Osland and A.R. Raklev, Gravitino Dark Matter and the Flavour Structure of R-violating Operators, Phys. Lett. B 677 (2009) 62 [arXiv:0811.2969] [INSPIRE].

[89] N.E. Bomark, S. Lola, P. Osland and A.R. Raklev, Photon, Neutrino and Charged Particle Spectra from R-violating Gravitino Decays, Phys. Lett. B 686 (2010) 152 [arXiv:0911.3376] [INSPIRE].

[90] I. Affleck, M. Dine and N. Seiberg, Dynamical Supersymmetry Breaking in Supersymmetric QCD, Nucl. Phys. B 241 (1984) 493 [INSPIRE]. 